\title{
Review
}

Ming Guo ${ }^{1}$, Jingfeng Zhang ${ }^{2 \star}$

\section{Advances in research on influencing factors of hepatitis B virus infection caused by blood transfusions}

DOI: 10.1515/ii-2017-0153

Received August 23, 2017; accepted September 9, 2017; published online November 28, 2017

\begin{abstract}
As one of the main factors affecting safe blood transfusion, hepatitis B virus (HBV) infection through blood transfer seriously endangers human health. Therefore, studies should focus on both reducing infection rate of $\mathrm{HBV}$ and accurately evaluating the risk of infection. This study discusses the main factors affecting HBV infection that results from blood transfusions, with the aim of gaining insights into reducing HBV infection.
\end{abstract}

Keywords: blood transfusion, hepatitis B virus, infectivity

\section{Introduction}

Hepatitis B virus (HBV) infection is one of the most serious problems that endanger human health. More than one-third of the population in the world were once infected with HBV, with $>360$ million translated into chronic infection [1]. Every year, approximately 62 million people die of fulminant hepatitis, cirrhosis, and liver cancer caused by HBV infection. A total of 4.5 million new infections have also been documented, with one-quarter developing into liver disease [2]. Blood transfusion is an important route of transmission for HBV infection. According to a report from the World Health Organization (WHO) Viral Hepatitis Secretariat, HB can be transmitted through unsafe blood products, resulting in risks that reach as high as $70 \%$ [3]. Therefore, studies must evaluate and reduce transfusion-related HBV infection. The following sections summarize the factors influencing HBV infection through blood transfusions and the recent relevant theories and practices of reducing blood transfusion-related HBV infection and ensuring safe blood transfer.

\section{$2 \mathrm{HBV}$ is the Most Important Factor Affecting Blood Transfusion Infectivity}

\subsection{Effects of HBV Genotype}

According to their entire gene sequences, differences among various HBV strains reach $>8 \%$, and to date, 10 genotypes (from A to J) have been discovered. Evidence indicates that different HBV genotypes lead to

\footnotetext{
${ }^{1}$ Department of Hematology, ${ }^{2}$ Department of Nephrology, Second Affiliated Hospital, Beijing University of Traditional Chinese Medicine, Beijing 100078, China

${ }^{*}$ Correspondence: Jingfeng Zhang, Department of Nephrology, Second Affiliated Hospital, Beijing University of Traditional Chinese Medicine, Beijing 100078, China. E-mail: xfsailors@sina.com
} 
different HBV infectivities through blood transfusions. Using experiments on gorillas infected with HBV, the American group Hsia et al. [4] proved that for different genotypes, 50\% chimp infectious dose $\left(\mathrm{CID}_{50}\right)$ of HBV differs. The $\mathrm{C}$ genotype spans three genome equivalents (geq, D genotype totals 78 geq, and A genotype measures 169 geq; however, the doubling time (1.9 days) of the C genotype HBV is shorter than that of the A genotype (3.4 days) [5]. Therefore, when only genotypes are considered, the $C$ genotype may be more susceptible than the A genotype. After replication for the same period of time, the C genotype is easier to detect HBV than A genotype. Therefore, further studies are needed to identify the risks of transmission of different genotypes of HBV through blood transfusion. Compared with the D genotype, Europeans and Americans perform better at clearing the A genotype HBe antigen (HBeAg), and compared with the $\mathrm{C}$ genotype, serological conversion of the B genotype HBeAg occurs earlier [6]. Presence of HBeAg indicates active replication of HBV, and reduction in HBeAg levels or serological conversion signifies decline in viral replication. In different blood recipients, different genotypes of HBV exhibit varying replication activities. These genotypes also present different susceptibilities to blood transfusion-related HBV infection.

\subsection{Influences of HBV Variability}

Under natural conditions or due to drug effects or vaccination, mutation may occur in different parts of the HBV genome, leading to changes in the biological characteristics of the virus. Some changes affect risks of transfusion-related HBV infection. Changes in HBV antigen and antibody expression may include mutations in the loci in the S-region; they may alter the alpha determinant of genes in the S-region, thereby altering the antigenicity of HB surface antigen (HBsAg) of HBV and affecting its ability to bind to antibodies [7]. For different mutations of HBsAg, current enzyme-linked immunosorbent assay diagnostic reagents exhibit different detection capacities. Occasionally, missing detections transpire, leading to false negative results of $\mathrm{HBsAg}$ detection and increased risk of HBV transmission through blood transfusions. Changes in HBV replication activity may include mutations in T1753C, C1766T, and T1768A in the basic core promoter region of the $\mathrm{C}$ gene, and they can also improve the level of viral replication [8]. Therefore, under the same circumstances, when recipients are injected with $\mathrm{HBV}$ mutants that can improve the level of viral replication, HBV susceptibility may change. Changes in host immunoreactivity, such as mutations in the key codon of certain viral epitope peptides in the $\mathrm{C}$ gene, affect either the binding of the epitope peptide to human leukocyte antigen (HLA) or the identification of T-cell receptor on the epitope peptides, thereby preventing immune attacks [9]. When recipients are injected with $\mathrm{HBV}$-infected blood products containing such variants, risks of HBV infection may increase.

\subsection{Influence of HBV Infection Period}

HBV infection can be divided into different phases, namely, eclipse, pre-ramp-up, ramp-up, plateau, and postseroconversion phases. The virulence of virus particles differs in different phases, leading to varying degrees of risks of HBV infection. Animal experiments conducted by Tabuchi et al. [10] showed that when chimpanzee serum was used to infect laboratory mice with HBV, virulence in the early stage of acute infection was 100 times that of late-stage acute infection. In the early stage of acute infection, such as in the ramp-up phase, as the corresponding neutral antibodies were not yet produced, the HBV $\mathrm{CID}_{50}$ was 3-10 virus particles. In the late acute infection stages, specifically the decrement phase, $\mathrm{HBV} \mathrm{CID}_{50}$ comprised 300-1,000 virus particles. In reported HBsAg-/HBV DNA+ HBV-infected cases, infected virus load in the window period (WP) measured $10 \mathrm{IU} / \mathrm{mL}$. However, in the postseroconversion phase, viral load of occult HBV infection (OBI) was generally $>100 \mathrm{IU} / \mathrm{mL}$ and was related to $\mathrm{HB}$ core antibody (anti-HBc) titer and the presence/absence of HB surface antibodies (anti-HBs). In the presence of high-titer anti-HBc, the infection rate reached $17 \%$. In the presence of low-titer anti-HBc, the infection rate totaled $10 \%$, but the rate reached $1.3 \%$ when accompanied by anti-HBs [11]. 


\section{Blood Screening Strategies Controlling Risk of HBV Infection}

\subsection{Influence of Screening Strategies}

HBV particles present complex and varied serological characteristics. Different serum markers manifest in people infected with HBV at different periods. WHO recommends various HBV screening strategies, including HBsAg, anti-HBc (where appropriate), and virus nucleic acid testing (NAT) for HBV DNA [1]. Different countries must develop appropriate HBV screening strategies based on specific circumstances (such as virus prevalence) to ensure blood transfusion safety. Because of their own characteristics and local environmental conditions, different screening strategies feature different detection rates of HBV, affecting the infectivity of HBV transmission through blood transfusions to a certain extent. Currently, with technical progress, the sensitivity of HBsAg detection reaches $0.005 \mathrm{ng} / \mathrm{mL}$ [12]. HBV DNA detection is generally carried out simultaneously with detection of human immunodeficiency virus (HIV) RNA and hepatitis C virus RNA; the specificities of polymerase chain reaction (PCR)-based commercial detection and transcription-mediated amplification-based detection reach $99.9 \%$ and $99.8 \%$, respectively, with sensitivities of 8 and $12 \mathrm{IU} / \mathrm{mL}$ [12]. NAT minimizes the risk of infection through blood transfusions. According to the study by the Working Party on Transfusion-Transmitted Infectious Diseases in 59 countries and regions after implementation of NAT, among 1,098,306 specimens, 75 were in the WP, accounting for 6.8/1,000,000 of all specimens, and 250 presented OBI, accounting for 22.8/1,000,000 of all specimens. In the past 10 years, a number of countries and regions have increased NAT testing [14]. Studies show that anti-HBc detection can reduce HBV transmission through blood transfusions, especially in terms of OBI reduction. In low-prevalence HBV areas, such as the United States and Canada, anti-HBc is listed as a regular test item, significantly improving the safety of blood transfusion [12]. Countries and regions with high prevalence of HBV promote highly sensitive HBsAg detection and NAT tests for individuals [15].

\subsection{Limitations of Blood Screening Methods}

Irrespective of whether HBsAg detection or the NAT test or a joint anti-HBc test is used, results do not fully reflect actual conditions, nor do they completely prevent HBV infection. All these methods feature some limitations, which are as follows: 1) for specimens of blood donors who are vaccinated against HB within a month, test results can be HBsAg-positive; 2) sensitivity of HBsAg detection is influenced by many factors, including virus genotype, mutations, presence of anti-HBsAg in blood donors, and coexistence of joint infection with other diseases [11]; 3) NAT test is divided into mini-pool (MP) NAT and individual donation (ID) NAT. Studies show that the HBsAg method requires 50-60 days to detect HBV infection, and MP NAT can shorten the time to 40-50 days; ID NAT requires 15-34 days to detect HBV infection [16]. However, NAT cannot detect HBV in the initial stage of infection; 4) anti-HBc detection is not applicable in HBV high-prevalence areas because it leads to huge loss of blood donors, resulting in insufficient blood supply; 5) for most anti-HB blood products $>100 \mathrm{IU} / \mathrm{mL}$, though they are anti-HBc(+) or contain HBV DNA and do not transmit HBV, we cannot rule out the possibility that anti-HB(+) blood products may cause HBV infection. Overall, even when HBsAg, HBV DNA, and anti-HBc tests are all negative, such results do not necessarily prevent HBV transmission, which is related to characteristics of the virus itself, immune status of recipients, and test limitations.

\section{Self-Condition of Recipients is Closely Related to HBV Infectivity}

\subsection{Effects of Immune Status of Recipients}

The immune status of recipients is closely related to their susceptibility to HBV. Compared with recipients with normal immune function, those with immune deficiency are at higher risk of HBV infection through 
blood transfusions. From studies on Mexican recipients, Calderon et al. [17] observed that when recipients exhibit conditions such as end-stage renal disease or the need for hemodialysis and organ transplantation, which affect their immune status, their risk of HBV infection (7\%) was higher than that of healthy donors $(0.13 \%)$. The HBV infection rate reached $13.2 \%$ for recipients with end-stage renal disease, $5.5 \%$ for recipients with cancer, and $2.4 \%$ for recipients with hemophilia. In China, local studies also confirmed higher HBV carrier rate of patients with blood diseases than the general population. Among these individuals, the carrier rate reached $31.37 \%$ for patients with aplastic anemia, $19.91 \%$ for patients with idiopathic thrombocytopenic purpura, and $16.9 \%$ for patients with leukemia; the control group of healthy people without blood disease presented a carrier rate of $10.12 \%$ [18]. According to the WHO, nearly $10 \%$ of AIDS patients are jointly infected with HBV. Recipients infected with HIV show decreased immune response to HBV infection and increased HBV replication. Therefore, their serum contains high HBV DNA levels, and the HBeAg clearance rate is reduced. However, viral replication barely reduces. Thus, susceptibility to HBV increases. When such recipients are transfused with blood products, HBV infection may occur [1].

\subsection{Influence of Genetic Background of Recipients}

The immunogenetic background of recipients, especially the HLA status, is one of the important factors that determine the possibility of infection with HBV. HLA plays a vital role in the immune identification of HBV, immune response, and immune regulation. Genetic polymorphism of this antigen is closely related to the susceptibility of different individuals to HBV. Distribution of some genes differs in populations and among different races. When associated with the safety of blood transfusion, this condition manifests as influences of HLA polymorphism on susceptibility of different individuals and recipients of different races to HBV; such influences include involvement of HLA-I genes in identification of CD8+ T-cells and the killing and dissolution of infected cells by inducing cytotoxic T-lymphocytes (CTLs). When the recipient CTLs present an inadequate HBV response, incomplete clearance of HBV may occur. Accordingly, the recipient is infected with HBV and develops chronic HBV infection. In the Chinese Taiwan population, the $H L A-A^{*} 0206$ gene, in the Chinese Han population, the HLA-B35 gene, in the Russian population, the HLA-B18, -B35, and -B40 genes, and in the Kazak population, the $H L A-A 3$ and -B18 genes are related with persistent HBV infection, whereas in the Taiwan population, the HLA-B6 gene and in the American Caucasian population, the HLA$A^{*} 030$ genes prevent such conditions [19,20]. HLA-II genes are involved in the identification of CD4+ T-cells; this phenomenon is related to the immune response and virus clearance after HBV infection. Studies show that HLA-DPA1*0202-DPB1*0501 and HLA-DPA1*0202-DPB1*0301 are positively correlated with the chronicity of HBV infection, whereas HLA-DPA1*0103-DPB1*0402 and HLA-DPA1*0103-DPB1*0401 may protect the body from long-term HBV infection [21].

\section{Vaccine is the Most Effective Way to Prevent HBV Infection}

As the most effective way to prevent $\mathrm{HBV}$ infection, $\mathrm{HB}$ vaccine plays an important role in reducing $\mathrm{HBV}$ infection related to blood transfusion. In 1992, the WHO proposed that by 1997 , all countries should incorporate $\mathrm{HB}$ vaccine into their planned immunization programs to eliminate HB worldwide after a few generations. Taiwan is one of the first regions in the world that started HB vaccination. In 1984, before HB vaccination, the HBsAg prevalence rate was 9.6\%, whereas in 1989, 1994, 1999, and 2004, after vaccination, the rates decreased to $4.6 \%, 1.4 \%, 0.7 \%$, and $0.4 \%$, respectively [22]. Incidence of hepatocellular carcinoma also reduced from $0.7 / 100,000$ in 1981-1986 to 0.36/100,000 in 1990-1994 [23]. Incidence rates of liver cancer in Italy, Japan, and Gambia reduced by $75 \%$ after vaccination [24]. In 1992, China incorporated the HB vaccine into immunization management in children. In 2002, China incorporated HB vaccination for all newborns into its national immunization program. Compared with the survey in 1992, the 2006 National HBV-Seroepidemiology Survey of Viral Hepatitis showed significantly decreased prevalence rate of HBsAg among 1- to 
14-year-old children, especially in children $<5$ years old. Prevalence rate reached $1.0 \%$, a decrease of $90 \%$ compared with that calculated in 1992 (9.8\%). In individuals between 15 and 19 years old, the prevalence rate of HBsAg also decreased [25]. These results showed that HB vaccine can effectively reduce both HBV infection rate and incidence rate of liver cancer. Thus, HBV carrier rate decreases accordingly in blood donors. As vaccinated recipients produce anti-HBs in their bodies, their risk of HBV infection also decreases. However, using $\mathrm{HB}$ vaccine also poses some limitations when using it to prevent $\mathrm{HBV}$ infection related with blood transfusions. Currently, infants, young people, and high-risk groups are the main targets of HB vaccination. However, in all populations, $5 \%-10 \%$ are healthy, and $>30 \%$ possess immune deficiency which shows absence of or low response to HB vaccine [9]; this observation is mainly related to genetics, immunity, and joint infection.

\section{Other Influencing Factors}

Different blood products result in varying risks of HBV transmission. Compared with whole blood, blood components exhibit low HBV infectivity. However, according to WHO 2011 global blood safety statistics, in low-income countries, only $31 \%$ of blood was fractionated, compared with $91 \%$ and $72 \%$ observed in highand middle-income countries, respectively [1]. Red blood cell suspensions contain approximately 20-40 mL of plasma, and once the contents of HBV DNA in the plasma reach over 1 geq/20 mL, HBV infection may transpire [11]. Compared with repetitive blood donors, first-time blood donors display higher risk of HBV infection. For example, in Africa, the detection rate of HBV DNA for first-time blood donors totals 120.93/100,000, whereas that for repetitive blood donors reaches 32.31/100,000; in the Asia-Pacific region and Europe, the corresponding numbers total 53.82/100,000 and 22.11/100,000; and 3.29/100,000 and 2.76/100,000, respectively [14]. Compared with paid blood donors, voluntary blood donors show much lower risk of HBV infection. According to a WHO report, the blood safety of voluntary blood donors is 5-10 times higher than that of paid donors. However, in 40 countries that participated in the survey, $<25 \%$ of blood supply came from voluntary blood donors. Thus, most blood supplies still depend on family/alternative blood donors and paid blood donors. In 2008, 26 countries were reported to rely on paid blood donation, and the total amount of paid blood donations was approximately 80 million times that of voluntary blood donors [1].

\section{Improvement Strategies and Methods of Reducing HBV Infection Caused by Blood Transfusions}

Diverse factors influence HBV infection caused by blood transfusions. Risks of blood transfusion-related HBV infection can be reduced by in-depth studies on these factors and applying joint targeted measures. Related strategies and methods include the following: 1) based on epidemic situation of local HBV genotypes, different countries and regions should develop specific HB vaccines that target the local population and strengthen vaccination for newborns and those who missed vaccinations; 2) monoclonal antibodies with two or more strains as capture antibodies and polyclonal antibodies must be used in detection methods to improve detection sensitivity of HBsAg and capability to detect variant strains; 3 ) blood must be collected from low-risk blood donors, unpaid blood donation should be implemented, NAT screening for blood must be implemented immediately, highly sensitive test reagents should be selected and developed, and small-sample mixtures and single-sample detection must be adopted;4) white blood cell filtration and virus inactivation should be popularized across regions, and clinical blood transfusion indications must be fully grasped while simultaneously adhering to the principle of transfusing only when needed. Overtransfusion and implementation of component transfusion should be avoided; 5) immune status of recipients must be considered during transfusion of blood products. For immune-deficient recipients, relevant treatments of blood products should be conducted, or immunoglobulin should be injected before transfusion. 


\section{Summary}

In the recent 20 years, although the safety of blood transfusion has remarkably improved, transfusionrelated HBV infection remains a significant threat to human health. Influencing factors not only include virus genotype and mutation, virus replication period, different screening strategies in different regions, specificity and sensitivity of detection method, immune status of recipient and ability to respond to vaccine, choice of appropriate blood donors, separation of blood components, virus inactivation and treatment, and other factors but also factors that still require definitive conclusions; these uncertain factors include volume of plasma infused, load of viruses existing in cells or bound with cells but escaping NAT detection, weight/ blood volume of recipient, storage time of blood products, and preservation temperature. Therefore, further studies must be performed to reduce transfusion-related HBV infection. Scholars from all over the world must also collaborate to explore and continually study and search for additional means of reducing risk of such conditions.

Authors' Contributions: M Guo made the literature analysis and wrote, discussed, and revised the manuscript of this review. JF Zhang critically analyzed and corrected the manuscript. All authors read and approved the final manuscript.

Acknowledgments: None.

Conflict of Interest: The authors state no conflict of interest.

\section{References}

[1] WHO, Hepatitis B vaccines, Weekly Epidemiol. Rec., 2009, 84(3), 405-420.

[2] Zanetti A.R., Van Damme P., Shouval D., The global impact of vaccination against hepatitis B: a historical overview, Vaccine, 2008, 26(12), 6266-6273.

[3] WHO, Report from the Secretariat on the Viral Hepatitis, Geneva, 63rd World Health Assembly, 2000-01-05 [2013-01-16], http://www.docin.com/p-289449237.html.

[4] Hsia C.C., Purcell R.H., Farshid M., Lachenbruch P.A., Yu M.Y., Quantification of hepatitis B virus genomes and infectivity in human serum samples, Transfusion, 2006, 46(10), 1829-1835.

[5] Komiya Y., Katayama K., Yugi H., Mizui M., Matsukura H., Tomoguri T., et al., Minimum infectious dose of hepatitis B virus in chimpanzees and difference in the dynamics of viremia between genotype A and genotype C, Transfusion, 2008, 48(2), 286-294.

[6] Lai C.L., Ratziu V., Yuen M.F., Poynard T., Viral hepatitis B, Lancet, 2003, 362(9401), 2089-2094.

[7] Servant-Delmas A., Mercier M., El Ghouzzi M.H., Girault A., Bouchardeau F., Pillonel J., et al., National survey of hepatitis B virus (HBV) polymorphism in asymptomatic HBV blood donors from 1999 to 2007 in France. Transfusion, 2010, 50(12), 2607-2618.

[8] Jammeh S., Tavner F., Watson R., Thomas H.C., Karayiannis P., Effect of basal core promoter and pre-core mutations on hepatitis B virus replication, J. Gen. Virol., 2008, 89(4), 901-909.

[9] Li P., Virus mutation, In: L. Kangxian, C. Jinjun, L. Ping (Ed.), Principles of Hepatitis B and Hepatitis B in Clinic, 4th ed., People's Health Publishing House, Beijing, 2012, 61-63.

[10] Tabuchi A., Tanaka J., Katayama K., Mizui M., Matsukura H., Yugi H., et al., Titration of hepatitis B infectivity in the sera of pre-acute and late acute phases of HBV infection: transmission experiments to chimeric mice with human liver repopulated hepatocytes, J. Med. Virol., 2008, 80(8), 2064-2068.

[11] Kleinman S.H., Lelie N., Busch M.P., Infectivity of human immunodeficiency virus-1, hepatitis C virus, and hepatitis B virus and risk of transmission by transfusion, Transfusion, 2009, 49(11), 2454-2489.

[12] Ye X., Liu X., Blood screening progress of hepatitis B virus, Chin. J. Blood Transfus., 2012, 25(7), 704-707.

[13] Candotti D., Allain J.P., Transfusion-transmitted hepatitis B virus infection, J. Hepatol., 2009, 51(4), 798-809.

[14] Roth W.K., Busch M.P., Schuller A., Ismay S., Cheng A., Seed C.R., et al., International survey on NAT testing of blood donations: expanding implementation and yield from 1999 to 2009, Vox Sang, 2012, 102(1), 82-90.

[15] Allain J.P., Occult hepatitis B virus infection: implications in transfusion, Vox Sang, 2004, 86(2), 83-91.

[16] Biswas R., Tabor E., Hsia C.C., Wright D.J., Laycock M.E., Fiebig E.W., et al., Comparative sensitivity of HBV NATs and HBsAg assays for detection of acute HBV infection, Transfusion, 2003, 43(6), 788-798. 
[17] Calderon G.M., Gonzalez-Velazquez F., Gonzalez-Bonilla C.R., Novelo-Garza B., Terrazas J.J., Martínez-Rodríguez M.L., et al., Prevalence and risk factors of hepatitis C virus, hepatitis B virus, and human immunodeficiency virus in multiply transfused recipients in Mexico, Transfusion, 2009, 49(10), 2200-2207.

[18] Liu Y., Ren Y., Su B., et al., Analysis of the relationship between hepatitis B virus infection and hematologic malignancies, Modern Oncology, 2011, 19(1), 165-166.

[19] Wu Y.F., Wang L.Y., Lee T.D., Lin H.H., Hu C.T., Cheng M.L., et al., HLA phenotypes and outcomes of hepatitis B virus infection in Taiwan, J. Med. Virol., 2004, 72(1), 17-25.

[20] Thio C.L., Thomas D.L., Karacki P., Gao X., Marti D., Kaslow R.A., et al., Comprehensive analysis of class I and class II HLA antigens and chronic hepatitis B virus infection, J. Virol., 2003, 77(22), 12083-12087.

[21] Kamatani Y., Wattanapokayakit S., Ochi H., Kawaguchi T., Takahashi A., Hosono N., et al., A genome-wide association study identifies variants in the HLA-DP locus associated with chronic hepatitis B in Asians, Nat. Genet., 2009, 41(5), 591-595.

[22] Hsu H.Y., Chang M.H., Ni Y.H., Chiang C.L., Chen H.L., Wu J.F., et al., No increase in prevalence of hepatitis B surface antigen mutant in a population of children and adolescents who were fully covered by universal infant immunization, J. Infect. Dis., 2010, 201(8), 1192-1200.

[23] Chang M.H., Chen C.J., Lai M.S., Hsu H.M., Wu T.C., Kong M.S., et al., Universal hepatitis B vaccination in Taiwan and the incidence of hepatocellular carcinoma in children. Taiwan Childhood Hepatoma Study Group, N. Engl. J. Med., 1997, 336(10), 1855-1859.

[24] Lavanchy D., Viral hepatitis: global goals for vaccination, J. Clin. Virol., 2012, 33(2), 296-300.

[25] Liang X., Bi S., Yang W., Wang L., Cui G., Cui F., et al., Epidemiological serosurvey of hepatitis B in China - declining HBV prevalence due to hepatitis B vaccination, Vaccine, 2009, 27(47), 6550-6557. 